\title{
Extension of application field of analytical formulas for the computation of projectile motion in midair
}

(Extensão do campo de aplicações de fórmulas analíticas para os cálculos do movimento de um projétil no ar)

\author{
Peter Chudinov ${ }^{\mathbb{m}}$ \\ Perm State Agricultural Academy, Perm, Russian Federation \\ Recebido em 12/4/2012; Aceito em 23/6/2012; Publicado em 18/2/2013
}

\begin{abstract}
The classic problem of the motion of a point mass (projectile) thrown at an angle to the horizon is reviewed. The air drag force is taken into account with the drag factor assumed to be constant. An analytical approach is used for the investigation. Application field of the previously obtained approximate analytical formulas has been expanded both in the upward launch angle, and in the direction of increase of the initial speed of the projectile. The motion of a baseball is presented as an example. It is shown that in a sufficiently wide ranges of initial velocity and launch angle the relative error in calculating the distance of the ball does not exceed $1 \%$.
\end{abstract} Keywords: spherical object, quadratic drag force, analytical formula, relative error.

O problema clássico do movimento de um ponto material (projétil) jogado com determinado ângulo em relação à direção horizontal é revisto nesse artigo. A força de resistência do ar é levada em conta, supondo que o coeficiente de arrastamento seja constante. Uma abordagem analítica é utilizada para essa investigação. O campo de aplicação das fórmulas analíticas aproximadas obtidas anteriormente foi expandido tanto em relação ao ângulo de lançamento quanto em relação aos valores da velocidade inicial do projétil. O movimento de uma bola de basebol é apresentado como exemplo. Mostra-se que, para intervalos suficientemente amplos de velocidade inicial e ângulo de lançamento, o erro no cálculo da distância percorrida pela bola não é superior a $1 \%$.

Palavras-chave: objeto esférico, força quadrática de arrastamento, fórmulas analíticas, erro relativo.

\section{Introduction}

The problem of the motion of a point mass (projectile) thrown at an angle to the horizon has a long history. The number of works devoted to this task is immense. It is a constituent of many introductory courses of physics. This task arouses interest of authors as before [1-6]. With zero air drag force, the analytic solution is well known. The trajectory of the projectile is a parabola. In situations of practical interest, such as throwing a ball, taking into account the impact of the medium the quadratic resistance law is usually used. In that case the problem probably does not have an exact analytic solution and therefore in most scientific publications it is solved numerically [7-11]. Analytic approaches to the solution of the problem are not sufficiently advanced. Meanwhile, analytical solutions are very convenient for a straightforward adaptation to applied problems and are especially useful for a qualitative analysis. Therefore the attempts are being continued to construct analytical solutions (even approximate) for this problem. Comparativly simple approximate ana-

\footnotetext{
${ }^{1}$ E-mail: chupet@mail.ru.

lytical formulas to study the motion of the point mass in a medium with a quadratic drag force have been obtained using such an approach [12-14]. These formulas make it possible to carry out a complete qualitative and quantitative analysis without using numerical integration of differential equations of point mass motion. The proposed analytical solution differs from other solutions by easy formulas, ease of use and acceptable accuracy. It is intended to study the motion of a baseball, and other similar objects.

Present article is largely initiated by the interesting work of Hackborn [4]. In this paper the accuracy of various analytical approximations of the projectile trajectories was investigated in the calculation of their distance in a fairly wide ranges of variation of the initial velocity and launch angle. The purpose of present paper is to extend the application field of the formulas [12-14] and to compare the accuracy of these formulas for calculating the projectile range with the results obtained in Ref. [4]. In the paper under consideration the term "point mass" means the center of mass of a smooth spherical object of finite radius $r$ and 
cross-sectional area $S=\pi r^{2}$. The conditions of applicability of the quadratic resistance law are deemed to be fulfilled, i.e. Reynolds number $R e$ lies within $1 \times 10^{3}<R e<3 \times 10^{5}[5]$.

\section{Equations of point mass motion and analytical formulas for basic param- eters}

Suppose that the force of gravity affects the point mass together with the force of air resistance $\boldsymbol{R}$ (Fig. 1), which is proportional to the square of the velocity of the point and directed opposite the velocity vector. For the convenience of further calculations, the drag force will be written as $R=m g k V^{2}$. Here $m$ is the mass of the projectile, $g$ is the acceleration due to gravity, $k$ is the proportionality factor. Vector equation of the motion of the point mass has the form

$$
m \boldsymbol{w}=m \boldsymbol{g}+\boldsymbol{R},
$$

where $\boldsymbol{w}$ - acceleration vector of the point mass. Differential equations of the motion, a commonly used in ballistics, are as follows [15]

$$
\begin{aligned}
& \frac{d V}{d t}=-g \sin \theta-g k V^{2}, \frac{d \theta}{d t}=-\frac{g \cos \theta}{V}, \\
& \frac{d x}{d t}=V \cos \theta, \frac{d y}{d t}=V \sin \theta .
\end{aligned}
$$

Here $V$ is the velocity of the point mass, $\theta$ is the angle between the tangent to the trajectory of the point mass and the horizontal, $x, y$ are the Cartesian coordinates of the point mass, $k=\frac{\rho_{a} c_{d} S}{2 m g}=$ const is the proportionality factor, $\rho_{a}$ is the air density, $c_{d}$ is the drag factor for a sphere, and $S$ is the cross-section area of the object (Fig. 1). The first two equations of the system (1) represent the projections of the vector equation of motion for the tangent and principal normal to the trajectory, the other two are kinematic relations connecting the projections of the velocity vector point mass on the axis $x, y$ with derivatives of the coordinates.

The well-known solution [15] of Eqs. (1) consists of an explicit analytical dependence of the velocity on the slope angle of the trajectory and three quadratures

$$
\begin{gathered}
V(\theta)=\frac{V_{0} \cos \theta_{0}}{\cos \theta \sqrt{1+k V_{0}^{2} \cos ^{2} \theta_{0}\left(f\left(\theta_{0}\right)-f(\theta)\right)},} \\
f(\theta)=\frac{\sin \theta}{\cos ^{2} \theta}+\ln \left(\tan \left(\frac{\theta}{2}+\frac{\pi}{4}\right)\right), \\
t=t_{0}-\frac{1}{g} \int_{\theta_{0}}^{\theta} \frac{V}{\cos \theta} d \theta, \quad x=x_{0}-\frac{1}{g} \int_{\theta_{0}}^{\theta} V^{2} d \theta, \\
y=y_{0}-\frac{1}{g} \int_{\theta_{0}}^{\theta} V^{2} \tan \theta d \theta .
\end{gathered}
$$

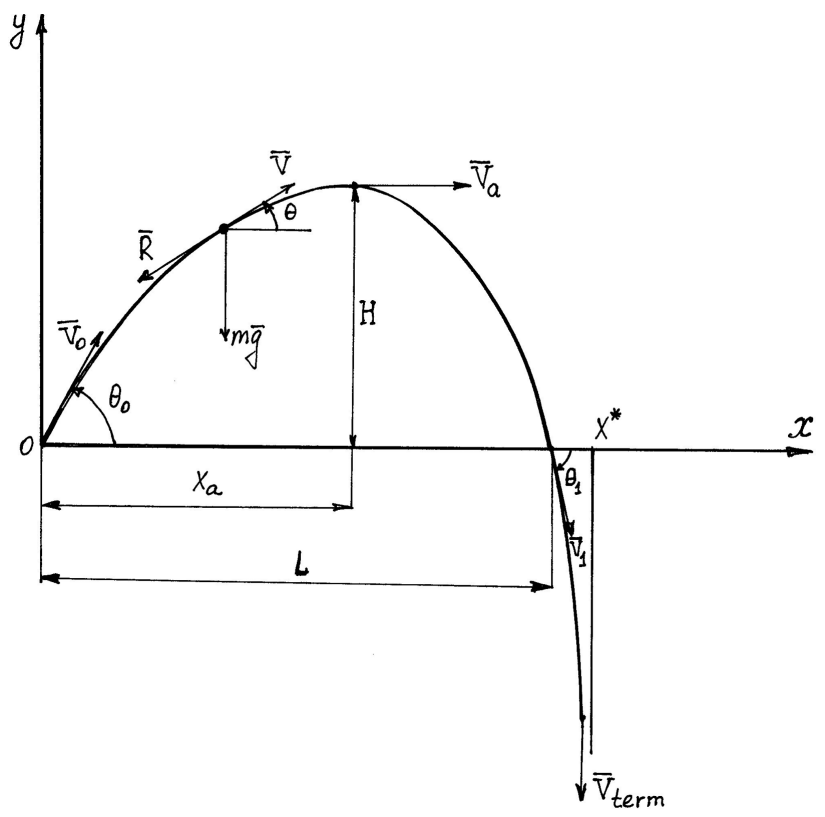

Figure 1 - Basic motion parameters.

Here $V_{0}$ and $\theta_{0}$ are the initial values of the velocity and the slope of the trajectory respectively, $t_{0}$ is the initial value of the time, $x_{0}, y_{0}$ are the initial values of the coordinates of the point mass (usually accepted $\left.t_{0}=x_{0}=y_{0}=0\right)$. The derivation of the formulas (2) is shown in the well-known monograph [16].

The integrals on the right-hand sides of Eqs. (3) cannot be expressed in terms of elementary functions. Hence, to determine the variables $t, x$ and $y$ we must either integrate Eqs. (1) numerically or evaluate the definite integrals (3). To avoid these procedures, comparatively simple approximate analytical formulas for the eight basic parameters of point mass motion were obtained in [12-14] (Fig. 1). The four parameters correspond to the top of the trajectory, four - to the point of drop. We will give a complete summary of the formulas for the maximum height of ascent of the point mass $H$, motion time $T$, the velocity at the trajectory apex $V_{a}$, flight range $L$, the time of ascent $t_{a}$, the abscissa of the trajectory apex $x_{a}$, impact angle with respect to the horizontal $\theta_{1}$ and the final velocity $V_{1}$

$$
\begin{gathered}
H=\frac{V_{0}^{2} \sin ^{2} \theta_{0}}{g\left(2+k V_{0}^{2} \sin \theta_{0}\right)}, \quad T=2 \sqrt{\frac{2 H}{g}}, \\
V_{a}=\frac{V_{0} \cos \theta_{0}}{\sqrt{1+k V_{0}^{2}\left(\sin \theta_{0}+\cos ^{2} \theta_{0} \ln \tan \left(\frac{\theta_{0}}{2}+\frac{\pi}{4}\right)\right)}} \\
L=V_{a} T, \quad t_{a}=\frac{T-k H V_{a}}{2}, \quad x_{a}=\sqrt{L H \cot \theta_{0}}, \\
\theta_{1}=-\arctan \left[\frac{L H}{\left(L-x_{a}\right)^{2}}\right], \quad V_{1}=V\left(\theta_{1}\right) .
\end{gathered}
$$

In formulas (4) $V_{0}$ and $\theta_{0}$ are the initial values of the velocity and the slope of the trajectory of the point 
mass, respectively. Formulas (4) enable us to calculate the basic parameters of motion of a point mass directly from the initial data $V_{0}, \theta_{0}$, as in the theory of parabolic motion. With zero drag $(k=0)$, formulas (4) go over into the respective formulas of point mass parabolic motion theory.

As an example of the use of formulas (4) we calculated the motion of a baseball with the following initial conditions

$$
\begin{gathered}
V_{0}=45 \mathrm{~m} / \mathrm{s} ; \theta_{0}=40^{\circ} ; \\
k=0.000548 \mathrm{~s}^{2} / \mathrm{m}^{2}, \mathrm{~g}=9.81 \mathrm{~m} / \mathrm{s}^{2} .
\end{gathered}
$$

The results of calculations are recorded in Table 1. The second column shows the values of parameters obtained by numerical integration of the motion Eqs. (1) with the fourth-order Runge-Kutta method. The third column contains the values calculated by formulas (4). The deviations from the exact values of parameters are shown in the fourth column of the table.

Table 1 - Numerical and analytical values of parameters.

\begin{tabular}{lccc}
\hline Parameter & Numerical value & Analytical value & Error (\%) \\
\hline$H, \mathrm{~m}$ & 30.97 & 31.43 & +1.5 \\
$T, \mathrm{~s}$ & 5.00 & 5.06 & +1.2 \\
$V_{a}, \mathrm{~m} / \mathrm{s}$ & 23.19 & 23.19 & 0.0 \\
$L, \mathrm{~m}$ & 117.8 & 117.4 & -0.3 \\
$t_{a}, \mathrm{~s}$ & 2.35 & 2.33 & -0.9 \\
$x_{a}, \mathrm{~m}$ & 65.36 & 66.32 & +1.5 \\
$\theta_{1}, \mathrm{deg}$ & -53.04 & -54.73 & +3.2 \\
$V_{1}, \mathrm{~m} / \mathrm{s}$ & 27.45 & 27.99 & +2.0 \\
\hline
\end{tabular}

\section{Extension of application field of the formulas (4)}

For baseball typical values of the drag force coefficient $k$ is about $0.0005 / 0.0006 \mathrm{~s}^{2} / \mathrm{m}^{2}[4,9]$. We introduce the notation $p=k V_{0}^{2}$. The dimensionless parameter $p$ has the following physical meaning - it is the ratio of air resistance to the weight of the projectile at the beginning of the movement. Formulas (4) have a bounded region of application. The main characteristics of the motion $H, T, V_{a}, L, t_{a}, x_{a}, \theta_{1}, V_{1}$ have accurate to within $2-3 \%$ for values of the launch angle, initial velocity and the parameter $p$ from ranges

$0^{\circ} \leq \theta_{0} \leq 70^{\circ}, \quad 0 \leq V_{0} \leq 50 \mathrm{~m} / \mathrm{s}, 0 \leq p \leq 1.5$.

We transform the formulas (4) so as to improve the accuracy of calculating the basic measure of the motion - range $L$ in the entire range of launch angles and at values of the initial velocity and the parameter $p$ larger than compared with the ranges of Eq. (5)

$$
0^{\circ} \leq \theta_{0} \leq 90^{\circ}, \quad 0 \leq V_{0} \leq 80 \mathrm{~m} / \mathrm{s}, \quad 0 \leq p \leq 4 .
$$

For this we consider the structure of the range formula $L=V_{a} T$. According to this formula, range of the motion is defined as the product of the velocity at the top of the trajectory $V_{a}$ on the time motion $T$ Therefore, to increase the accuracy of computation of the range $L$ it is necessary to increase the accuracy of calculating the parameters $V_{a}$ and $T$. Let's start with the increase the accuracy of the parameter $T$. In turn, according to the second of the formulas (4), the time of the projectile motion $T$ is determined by parameter $H$. The formula for the maximum height of ascent of the projectile $H$ is the most important of all the formulas (4). When the launch angle $\theta_{0}$ increases, height $H$ computed according to the first formula (4) is behind the exact value of this parameter. The exact value of $H$ can be obtained by integrating the equations of motion (1). The greatest noncoincidence occurs at an angle of throwing $\theta_{0}=90^{\circ}$. It is known [15] that the maximum height attained by the point mass at throwing with the initial conditions $V_{0}, \theta_{0}=90^{\circ}$, is given by

$$
H_{\max }=\frac{1}{2 g k} \ln \left(1+k V_{0}^{2}\right)
$$

Formula (4) for $H$ at $\theta_{0}=90^{\circ}$ gives the value of

$$
H_{1}=\frac{V_{0}^{2}}{g\left(2+k V_{0}^{2}\right)} .
$$

We introduce the notation

$$
\begin{gathered}
\Delta h=H_{\max }-H_{1}=\frac{1}{2 g k} \ln \left(1+k V_{0}^{2}\right)- \\
\frac{V_{0}^{2}}{g\left(2+k V_{0}^{2}\right)} .
\end{gathered}
$$

The quantity $\Delta h$ - is a mismatch between the exact and approximate values of the height at $\theta_{0}=90^{\circ}$. We form the function $f(p)$, equal to the ratio (8) to (7)

$$
f(p)=\frac{H_{1}}{H_{\max }}=\frac{2 p}{(2+p) \ln (1+p)} .
$$

The graph of $f(p)$ is shown in Fig. 2 .

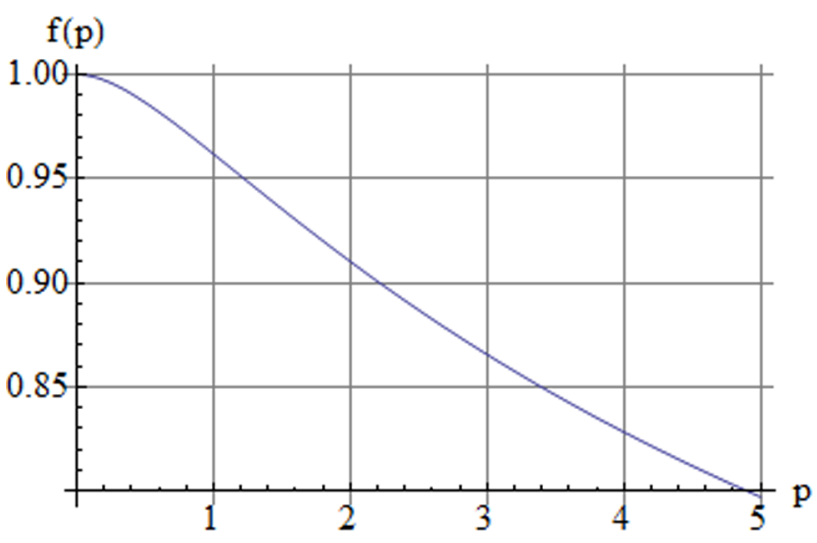

Figure 2 - Graph of the function $f=f(p)$. 
The graph shows that when the force of air resistance increases (parameter $p$ grows), formula (4) for $H$ loses accuracy when $\theta_{0}=90^{\circ}$. The same holds for large angles of throwing $\theta_{0} \geq 70^{\circ}$. To eliminate this drawback, we modify the formula (4) for $H$. Let it be like this

$$
H=\frac{V_{0}^{2} \sin ^{2} \theta_{0}}{g\left(2+k V_{0}^{2} \sin \theta_{0}\right)}+\Delta h \cdot f_{1}\left(\theta_{0}\right) .
$$

Here $f_{1}\left(\theta_{0}\right)$ is a function of the launch angle satisfying $f_{1}\left(0^{\circ}\right)=0, f_{1}\left(90^{\circ}\right)=1$. The choice of this function is quite arbitrary. Let the function $f_{1}\left(\theta_{0}\right)$ be given by the following empirical formula

$$
f_{1}\left(\theta_{0}\right)=\sin ^{4} \theta_{0}-\left(\frac{\theta_{0}}{90}\right)^{2} \cdot \cos ^{6} \theta_{0} .
$$

Graph of this function is shown in Fig. 3. Such a structure of formula (10) allows more accurate calculation of the height $H$ at high angles of throwing $\theta \geq 70^{\circ}$. When $\theta_{0}=90^{\circ}$, formula (10) gives the exact value of the height $(7)$.

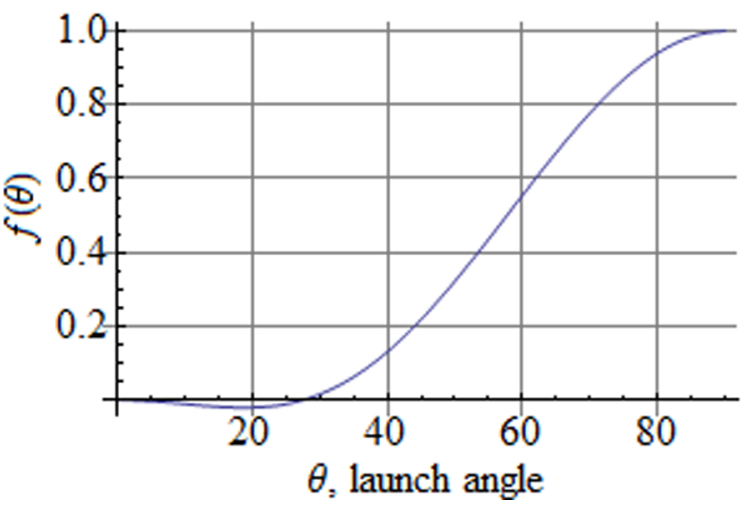

Figure 3 - Graph of the function $f_{1}=f_{1}\left(\theta_{0}\right)$.

Now we transform the second factor $V_{a}$ in the range formula. The parameter $V_{a}$ is the velocity of the projectile at the top of the trajectory calculated by the formulas (2) at $\theta=0^{\circ}$. Instead of the values of the velocity at the top of the trajectory at $\theta=0^{\circ}$ we calculate the velocity of the projectile at some close point of the trajectory defined by the angle of inclination $\theta_{a}$. We define angle $\theta_{a}$, measured in degrees, as a function of the parameters $p$ and $\theta_{0}: \theta_{a}=f_{2}\left(p, \theta_{0}\right)$. The choice of this function is arbitrary. However, the value of $\theta_{a}$ should be positive in order to increase the velocity of the projectile compared with the value of it at the top of the trajectory. This follows from the well-known fact that on the upward trajectory velocity is greater than at the top. We define the function $f_{2}$ as follows

$$
\theta_{a}=f_{2}\left(p, \theta_{0}\right)=p \cdot \frac{\pi}{180} \cdot \frac{\theta_{0}}{90} \cdot\left(1+2 \cdot \sin 2 \theta_{0}\right)
$$

Now under $V_{a}$ we understand the value determined by the relation

$$
V_{a}=V\left(\theta_{a}\right)
$$

Thus, the formulas (4) for $H$ and $V_{a}$ are replaced by the formulas (10), (12). In the absence of resistance of the medium $(k=0)$ formulas (10), (12) turn into the corresponding formulas of the parabolic motion theory. In addition, formulas (4) make it possible to obtain simple approximate analitical expressions for the basic functional relationships of the problem $y(x), y(t)$, $y(\theta), x(t), x(\theta), t(\theta)[13]$. For example, the trajectory equation $y(x)$ has the form

$$
y(x)=\frac{H x(L-x)}{x_{a}^{2}+\left(L-2 x_{a}\right) x} .
$$

This formula shows that for the construction of dependence $y(x)$ we need to know three parameters: $H$, $L$ and $x_{a}$, which are determined by formulas (4). This dependence $y(x)$ provides a shift of apex of the path to the right and has a vertical asymptote, as it is in case of the of projectile trajectories in the air. In the absence of the resistance $L=2 x_{a}$ and formula (13) go into the corresponding formula of the theory of parabolic motion.

\section{The results of calculations and their comparison}

The formulas (10), (12), (13) were used to calculate the motion of a baseball. It is convenient to calculate the coefficient of resistance $k$ in these formulas by means of the terminal velocity of a ball (Fig. 1): $k=1 / V_{\text {term }}^{2}$. We used the following typical values of $k$ (for $V_{\text {term }}=40 \mathrm{~m} / \mathrm{s}$ ) and the acceleration of gravity $g$

$$
k=\frac{1}{40^{2}}=0.000625 \mathrm{~s}^{2} / \mathrm{m}^{2}, \quad g=9.81 \mathrm{~m} / \mathrm{s}^{2} .
$$

The initial velocity, launch angle and parameter $p$ varied in the ranges (6). When calculating the range of the ball, the relative error was computed as a function of initial velocity and launch angle. Distance calculated according to formulas (4), (10), (12), was compared with the exact value of this parameter. The exact values of range were obtained by integration of the equations of motion (1) by the fourth-order Runge-Kutta method. The calculation results are shown in Fig 4. This figure is the main result of this article. 


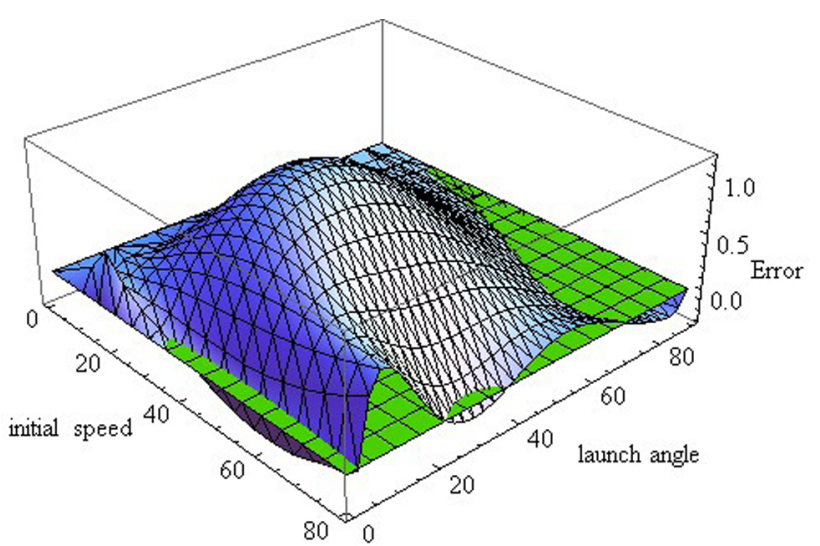

Figure 4 - The relative error in calculating the distance (in \%).

The horizontal plane in Fig 4 corresponds to the zero value of the relative error. Figure 4 shows that in the covered scope (6) the relative error is less than $1 \%$. Figure 5 shows the projectile trajectory $y(x)$ for values of $V_{0}=80 \mathrm{~m} / \mathrm{s}, \theta_{0}=60^{\circ}$. The solid line is obtained by integrating Eqs. (1), the broken line is constructed by formulas (4), (10), (12), (13). It is obvious that these formulas approximate a precise trajectory quite well. In paper [4] well-known analytical approximations of the trajectory of Parker [7], Littlewood [17], Lamb [18] are quoted, and also Hackborn gave his original formula, close to above-mentioned ones. These approximations were used in [4] to calculate the distance of baseball motion in the ranges of initial velocity and launch angle $10 \leq V_{0} \leq 80 \mathrm{~m} / \mathrm{s}, 15^{\circ} \leq \theta_{0} \leq 75^{\circ}$ for values of Eq. (14). The relative error in calculating the distance for these approximations was calculated in Ref. [4]. It was found by Hackborn that in these ranges the relative error reaches $4.5 \% \div 6.5 \%$. These values are much higher than the accuracy of $1 \%$ obtained with the help of formulas (4), (10), (12), (13).

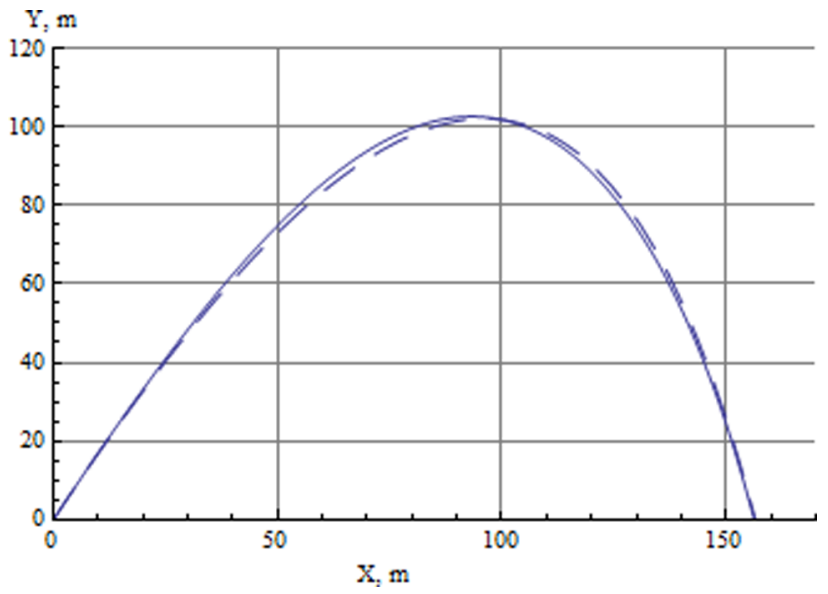

Figure 5 - Graph of the function $y=y(x)$.

\section{Conclusions}

The proposed modification of the formulas [12-14] allows us to significantly expand the range of their use in the studying of the projectile motion in midair. All the basic parameters of the motion and functional dependencies are still described by simple analytical relations. In addition the numerical values of the required quantities are determined with high accuracy. Thus, the formulas (4), (10), (12), (13) make it possible to study the motion of a point mass in a medium with resistance in the way it is done for the case of no drag.

\section{References}

[1] G.F.L. Ferreira, Revista Brasileira de Ensino de Física 23, 271 (2001).

[2] A.D.S. Bruno and J.M.O. Matos, Revista Brasileira de Ensino de Física 24, 30 (2002).

[3] E.N. Miranda, S. Nikolskaya and R. Riba, Revista Brasileira de Ensino de Física 26, 125 (2004).

[4] W. Hackborn, Canadian Applied Mathematics Quarterly 14, 285, (2006)

[5] A.Vial, European Journal of Physics 28, 657 (2007).

[6] J. Benacka, International Journal of Mathematical Education in Science and Technology 41, 373 (2010).

[7] G. Parker, American Journal of Physics 45, 606 (1977).

[8] H. Erlichson, American Journal of Physics 51, 357 (1983).

[9] A. Tan, C. Frick and O. Castillo, American Journal of Physics 55, 37 (1987).

[10] C. Groetsch, American Journal of Physics 65, 797 (1997).

[11] J. Lindemuth, American Journal of Physics 39, 757 (1971).

[12] P. Chudinov, International Journal of Mathematical Education in Science and Technology 41, 92 (2010).

[13] P. Chudinov, International Journal of Sports Science and Engineering 5, 27 (2011).

[14] P. Chudinov, European Journal of Physics 25, 73 (2004).

[15] B. Okunev, Ballistics (Voyenizdat, Moscow, 1943).

[16] S. Timoshenko and D. Young, Advanced Dynamics (McGraw-Hill Book Company, New York, 1948).

[17] J. Littlewood, Mathematical Spectrum 4, 80, (1971).

[18] H. Lamb, Dynamics (Cambridge University Press, London, 1923). 